\section{Global consensus building and prioritisation of fundamental lupus challenges: the ALPHA project}

Susan Manzi, ${ }^{1}$ Sandra Raymond, ${ }^{\odot}{ }^{2}$ Karin Tse, ${ }^{2}$ Yaritza Peña, ${ }^{3}$ Annick Anderson, ${ }^{3}$ Kathleen Arntsen, ${ }^{4}$ Sang-Cheol Bae, ${ }^{5}$ Ian Bruce, ${ }^{6}$ Thomas Dörner, ${ }^{7}$ Kenneth Getz, ${ }^{3}$ Leslie Hanrahan, ${ }^{2}$ Amy Kao, ${ }^{8}$ Eric Morand, ${ }^{9}$ Brad Rovin, ${ }^{10}$ Laura Eve Schanberg, ${ }^{11}$ Joan M Von Feldt, ${ }^{12}$ Victoria P Werth, ${ }^{13}$ Karen Costenbader ${ }^{14}$

To cite: Manzi S, Raymond S, Tse K, et al. Global consensus building and prioritisation of fundamental lupus challenges: the ALPHA project. Lupus Science \& Medicine 2019;6:e00342. doi:10.1136/ lupus-2019-000342

Received 22 May 2019 Revised 24 June 2019 Accepted 29 June 2019

Check for updates

(c) Author(s) (or their employer(s)) 2019. Re-use permitted under CC BY-NC. No commercial re-use. See rights and permissions. Published by BMJ.

For numbered affiliations see end of article.

Correspondence to

Sandra Raymond; raymond@ lupus.org

\section{ABSTRACT}

Objective Lupus is a complex, heterogeneous autoimmune disease that has yet to see significant progress towards more timely diagnosis, improved treatment options for short-term and long-term outcomes, and appropriate access to care. The Addressing Lupus Pillars for Health Advancement (ALPHA) project is the first step in establishing global consensus and developing concrete strategies to address the challenges limiting progress.

Methods A Global Advisory Committee of 13 individuals guided the project and began barrier identification. Seventeen expert interviews were conducted to further characterise key barriers. Transcripts were analysed using Nvivo and a codebook was created containing a list of thematic 'nodes' (topics) and their descriptions. Findings were used to develop a final survey instrument that was fielded to a diverse, international stakeholder audience to achieve broad consensus.

Results Expert interviews identified lupus heterogeneity as the primary barrier hindering advancement. Subsequent barriers were categorised into three areas: (1) Drug development. (2) Clinical care. (3) Access and value. The global survey received 127 completed responses from experts across 20 countries. Respondents identified barriers as high priority including the lack of biomarkers for clinical and drug development use, flawed clinical trial design, lack of access to clinicians familiar with lupus, and obstacles to effective management of lupus due to social determinants of care. Respondents also identified 30 autoimmune conditions that may be lupus-related based on overlapping features, shared autoantibodies and pathophysiology.

Conclusions ALPHA is a comprehensive initiative to identify and prioritise the continuum of challenges facing people with lupus by engaging a global audience of lupus experts. It also explored views on lupus as a spectrum of related diseases. Conclusions from this effort provide a framework to generate actionable approaches to the identified high-priority barriers.

\section{INTRODUCTION}

Lupus is a complex, heterogeneous autoimmune disease with enormous impact, disparities in outcomes and increased mortality. While lupus impacts people of all ages and races, it most often threatens the health of young women, who are often of non-Caucasian ethnicity and of low socioeconomic status. Among chronic inflammatory diseases in young women aged 15-24 years, it is the leading cause of death, with higher rates of death than diabetes mellitus or HIV. ${ }^{1}$ The prevalence of lupus is on the rise globally, and mortality rates from lupus remain three times as high as the general population. ${ }^{2}$ While lupus mortality rates declined for the last few decades of the 1900 s, they have been stagnant since, and enormous health disparities persist among adult and childhood lupus populations. Racial/ethnic groups, including African Americans, Hispanics and Asians have significantly higher risk of death due to lupus, ${ }^{3}$ and geographical differences, poverty and education levels, and insurance status also have a high impact on lupus mortality. ${ }^{34}$ There is notable impact on work, finances and other socioeconomic disparities associated with lupus. ${ }^{4-6}$ Not surprisingly, numerous healthcare access issues also exist, including access to specialty care and hospitals skilled in treating lupus. ${ }^{78}$ Such inequalities contribute to the significant unmet need in the care of people with lupus.

In contrast to other autoimmune diseases such as rheumatoid arthritis and psoriasis, approved medications for lupus remain very limited. Only one treatment, belimumab, has been developed specifically for lupus and has received regulatory approvals in the past 60 years. The field has long struggled with establishing a clear pathway for lupus drug development given persistent challenges in understanding the biology of the disease, defining clinical trial entry criteria and end points, developing instruments to measure 
changes in clinical activity, and controlling background medications. ${ }^{9}$ There have been repeated failures over the years to demonstrate superior drug efficacy compared with standard therapy, but optimistically, over 30 companies are currently investing in lupus clinical trials. ${ }^{10}$ Another consideration is safety. Some of the current treatments used for lupus, including glucocorticoids and immunosuppressive treatments, are associated with significant toxicity and poor long-term outcomes. ${ }^{11} 12$

\section{Prior barriers initiatives}

The Addressing Lupus Pillars for Health Advancement (ALPHA) project builds on the work of other important initiatives that have been more singular in focus, some of which have been international in scope or are still ongoing. In 2009, the Lewin Group conducted a series of lupus expert interviews and a panel discussion of industry, federal government and academic representatives focused on characterising barriers to drug development, which included use of confounding background medications in clinical trials, severe side effects of current treatment options, and lack of reliable and specific biomarkers for lupus. ${ }^{9}$ A 2019 landscape analysis outlines numerous insights learnt from clinical trials to date, yet many of the same barriers still exist a decade later. ${ }^{13} 14$ In the area of clinical care, an international task force involving various specialists and patient representatives began to address the concept of treat-to-target in lupus, developing an extensive research agenda. ${ }^{15}$ This initiative is ongoing, and has led to the creation of a framework for defining remission in lupus. ${ }^{16}$ Additionally, research on achieving low disease activity as a clinically meaningful treatment target also shows promising progress. ${ }^{17}$ These efforts have produced important results that underscore the success of coordinated efforts that engage multistakeholder audiences. To hasten progress and ensure the most urgent needs in lupus are being met, we undertook an initiative to tie together disparate projects and identify priorities common to global lupus research, care and access.

\section{The ALPHA project}

The ALPHA project was founded as the first step in an ongoing commitment to identify, prioritise and implement strategies to address the most pressing challenges that limit progress in lupus across the continuum. The ALPHA project was established as a comprehensive effort to engage a global audience of lupus experts to identify barriers impacting drug development, clinical care and access to care across the globe. ALPHA also began to explore how lupus is defined and how that influences major challenges in the field. The ultimate goal is to develop and implement strategies to address the key barriers to improving outcomes in lupus.

\section{METHODS}

A multiphase and mixed-methods approach was used to identify and validate consensus among global experts on the barriers to lupus diagnosis, care, treatment and research.

\section{The Global Advisory Committee}

The Lupus Foundation of America (LFA) and the Tufts Centre for the Study of Drug Development (Tufts CSDD) collaboratively identified the criteria for establishing a geographically and professionally diverse Global Advisory Committee (GAC) of lupus thought leaders to guide and oversee the study. Primary selection criteria included thought leadership in the field; extensive knowledge of the disease; global positioning; and ability to be a team player. The GAC identified a preliminary list of fundamental barriers, shaped the content for subsequent research instrument development, and provided overall direction throughout the study.

The GAC included 13 lupus experts across five countries (USA, Australia, UK, Germany and South Korea) representing clinician, scientist, academic researcher, biopharmaceutical industry, patient advocacy organisation and patient perspectives. GAC areas of expertise included rheumatology, dermatology, immunology, nephrology and paediatrics.

\section{In-depth expert interviews}

In-depth expert interviews were conducted to elicit further opinions and detailed rationale for most urgent barriers (including those identified by the GAC), assess the level of agreement across the lupus expert community, and inform content and design of the final survey instrument. LFA, Tufts CSDD and the GAC collaboratively identified interview candidates who were well respected by the lupus scientific and care communities; represented all stakeholders in the scientific community and industry; published relevant lupus articles within the past $2-5$ years (for researchers only); were currently providing direct treatment to people with lupus and/or were currently conducting lupus-related clinical research (for clinicians and principal investigators only); or had direct experience managing/helping others manage the disease, and were well-versed in current issues/knowledge of the lupus landscape (for patients/caregivers only).

Seventeen experts were interviewed. Interviewees resided in three different countries (USA, Japan and Netherlands) and represented academia, industry, regulatory agencies, clinicians, researchers, patient advocacy organisations and patients. Disease specialties included rheumatology, paediatrics, immunology, dermatology and musculoskeletal disease. Interviews were conducted by telephone and lasted approximately 1 hour. Interviewees provided their consent to participate and be audio-recorded using WebEx. Recordings were subsequently transcribed for analysis, summarised in Microsoft Excel and analysed using the qualitative analysis software, Nvivo, in which a codebook was created containing a list of thematic 'nodes', or topics, and their descriptions. Tufts CSDD conducted interrater assessments to ensure coding reliability. Nodes categorised related comments under subheadings to reveal 
emerging patterns and ideas. Nodes were also organised by the number of agreements and disagreements for each barrier. Barriers were then prioritised based on the highest frequency of mentions and agreements.

\section{Survey assessment}

Tufts CSDD, LFA and GAC collaborated to develop and refine the survey instrument. The final survey consisted of 23 questions focused on attitudes and perceptions about lupus and prioritisation of the most pressing challenges to improving diagnosis, care, treatment and research. Participants were asked to rank primary obstacles according to their relative impact on delayed lupus drug development, inaccurate or delayed diagnoses, suboptimal clinical care and delayed access to care, as well as identify lupus-related disorders. Survey respondents were solicited from prior expert lists developed while identifying GAC and interview candidates, and expanded based on the same criteria for in-depth interviews. The online survey was programmed through Qualtrics survey software and distributed over a 6-week period beginning November 2018.

Survey responses were cleaned in Microsoft Excel and imported into the Statistical Analysis Software program V 9.4. Descriptive statistics, frequency comparisons, subgroup stratification and weighted scoring were performed. Subgroup analyses used $\chi^{2}$ tests to look for significant differences by geography (US vs non-US), years of experience in the field and time spent in direct care for individuals with lupus. Significance was defined at the $\mathrm{p}<0.05$ level.

A weighting system was applied to prioritise barriers ranked by survey respondents depending on the frequency of being placed first, second or third in the high, medium and low priority categories. Higher ratings represented challenges of highest impact, while lower ratings represented those of lower impact. The highest possible rating was 9 and the lowest was 1 . For categories with less than nine barriers able to be ranked, a rating of 1-9 was still applicable given the limitations of forced-response programming on Qualtrics ranking questions. The weighted prioritisation score followed the algorithm:

\section{$\mathrm{X}_{\underline{1}} \mathrm{~W}_{1}+\mathrm{X}_{\underline{2}} \mathrm{~W}_{\underline{2}}+\ldots$ \\ Total response count}

\section{$\mathbf{X}=$ response count for answer choice$$
W=\text { weight of ranked position }
$$

Each phase of the research study was reviewed and deemed exempt by the Tufts Health Sciences Institutional Review Board.

\section{RESULTS}

Lupus heterogeneity and the lack of a clear disease definition were identified as the primary barriers causing the greatest challenge in development of clinically meaningful treatments, advancing clinical care, and providing improved access and value to patients. A strong consensus built throughout the study, as themes and insights gathered from the in-depth interviews were highly consistent with those collected in the survey.

The global online survey was distributed to 366 candidates and 127 (35\%) completed responses were received. Survey respondents had an average of 21 years working in the field, with several having more than 40 years of experience. When asked to identify their primary role, the majority $(82,65 \%)$ were clinician-researcher-scientists, $14(11 \%)$ were in industry/biotechnology, $13(10 \%)$ were researcher-scientists and $12(9 \%)$ were clinicians. Respondents were employed in a range of organisations including academic medical institutions $(99,78 \%)$, pharmaceutical/biotechnology companies $(13,10 \%)$, hospitals $(6,5 \%)$, private practice $(2,2 \%)$ and clinics $(1,1 \%)$. Other organisations $(6,5 \%)$ included regulatory agencies or biopharmaceutical consulting companies. Additionally, $81(64 \%)$ of respondents worked in USA and 46 $(36 \%)$ worked outside of USA. (table 1 ).

The survey results validated primary obstacles identified in earlier phases of the study. Survey respondents ranked the following as the top barriers to improving outcomes in lupus (table 2):

- Lack of diagnostic, predictive and prognostic biomarkers for lupus and lack of biomarkers to predict drug response in clinical trials.

- Flawed clinical trial design.

- Lack of access to clinicians familiar with lupus/ limited awareness of lupus among non-expert medical professionals.

- Barriers to effective management of lupus due to social determinants of care in predominantly lower socioeconomic status areas.

- Lack of treatment adherence.

Consensus was clear for items such as lack of biomarkers across clinical care and drug development; 93 (73\%) respondents ranked this high priority for clinical care and $74(58 \%)$ respondents for drug development. However, in both areas, $117(92 \%)$ and $114(90 \%)$ respondents, respectively, placed this in either high or medium priority categories. Additionally, flawed trial design was placed in the high priority category by $76(60 \%)$ respondents, which was the highest response of all drug development barriers. Lack of treatment adherence received a similar response under clinical care with $78(61 \%)$ responses indicating high importance. Barriers to effective management of lupus due to social determinants of care in low socioeconomic areas and lack of access to clinicians familiar with lupus were top-ranking barriers in the access and value areas, with 114 (90\%) respondents placing either in high or medium categories.

No significant differences were observed in the subgroup analyses conducted by geography, years of experience or time spent in direct care for individuals with lupus, largely due to the low number of subgroup 


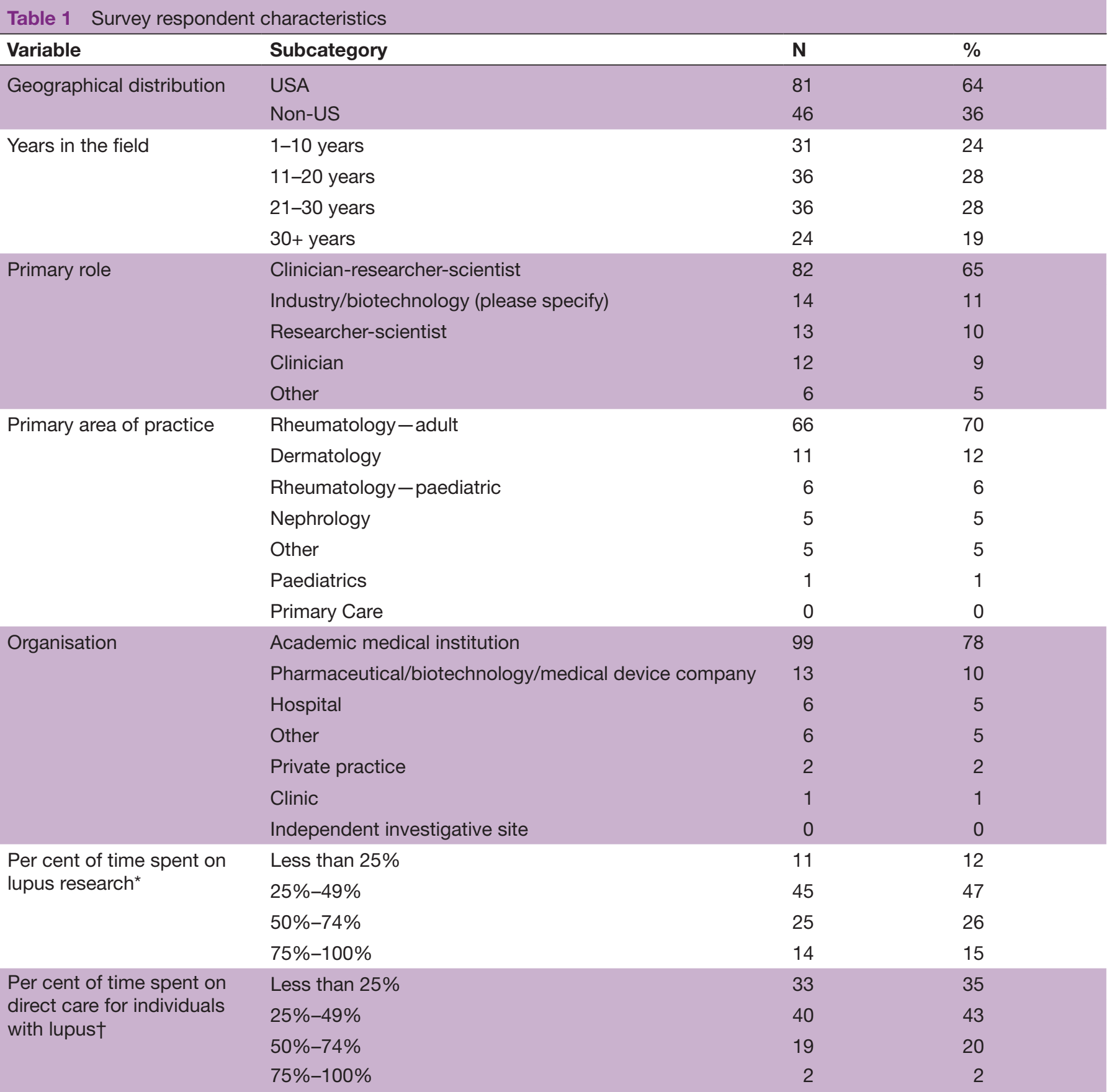

${ }^{*}$ Researcher-scientist or clinician-researcher-scientist.

†Clinician or clinician-researcher-scientist.

responses. However, respondents within each subgroup still ranked the same top three barriers in each area of drug development, clinical care, and access and value, validating agreement despite location or experience level.

Respondents identified 30 diseases, or disease terminologies, that were related to lupus, or 'fell under the lupus umbrella,' emphasising the complex aetiology and heterogeneity of the disease and the lack of a clear consensus definition. The three most frequently mentioned disease terminologies were lupus nephritis, cutaneous lupus and antiphospholipid antibody syndrome (APS).

\section{DISCUSSION}

The ALPHA project examined barriers that individually impact drug development, clinical care, and access and value, and the commonality across categories to assess the overall level of impact and ability to improve the quality of life of people with lupus. Heterogeneity of the disease was the primary barrier identified. Lack of validated biomarkers and flawed clinical trial design were among the highest prioritised barriers, as were lack of access to medical professionals familiar with lupus, challenges in managing lupus due to social determinants, and lack of treatment adherence. 
Table 2 Top barriers by category listed with their respective weighted ranked scores

\begin{tabular}{ll}
\hline Drug development & $\begin{array}{l}\text { Weighted } \\
\text { Prioritisation Score }\end{array}$ \\
\hline $\begin{array}{l}\text { Lack of biomarkers to predict response to } \\
\text { drug in clinical trials }\end{array}$ & 6.614 \\
$\begin{array}{l}\text { Lack of user-friendly, sensitive and accurate } \\
\text { outcome measures }\end{array}$ & 6.394 \\
$\begin{array}{ll}\text { Flawed clinical trial design } \\
\begin{array}{l}\text { Challenges with collecting patient-reported } \\
\text { and clinician-reported outcomes }\end{array}\end{array}$ & 6.370 \\
$\begin{array}{l}\text { Lack of alignment between patients, } \\
\text { providers and regulatory }\end{array}$ & 5.370 \\
\hline
\end{tabular}

Challenges with attracting under-represented 5.173 study populations

\begin{tabular}{ll}
\hline $\begin{array}{l}\text { Limited awareness of lupus among non- } \\
\text { expert medical professionals }\end{array}$ & 3.370 \\
\hline $\begin{array}{ll}\text { Lack of attention to R\&D paediatric issues } \\
\text { Lack of classification criteria }\end{array}$ & 3.173 \\
\hline $\begin{array}{l}\text { Clinical care } \\
\text { Lack of diagnostic, predictive and prognostic }\end{array}$ & $\begin{array}{l}\text { Weighted } \\
\text { Prioritisation Score }\end{array}$ \\
\hline $\begin{array}{l}\text { biomarkers for lupus } \\
\text { Lack of treatment adherence }\end{array}$ & 6.717 \\
\hline $\begin{array}{l}\text { Limited awareness and understanding } \\
\text { of lupus among non-expert medical } \\
\text { professionals }\end{array}$ & 5.800 \\
\hline $\begin{array}{l}\text { Lack of clear risk factors that trigger lupus } \\
\text { and lupus-like diseases (onset and flares) }\end{array}$ & 5.690 \\
\hline $\begin{array}{l}\text { Inadequate understanding of benefit-risk } \\
\text { trade-offs between physicians and patients }\end{array}$ & 5.103 \\
\hline \begin{tabular}{l} 
Lack of attention to paediatric issues \\
\hline $\begin{array}{l}\text { Lack of diagnostic criteria } \\
\text { payers/insurers about lupus and the }\end{array}$
\end{tabular} & 3.968 \\
\hline $\begin{array}{l}\text { Laccess and value } \\
\text { evidence }\end{array}$ & 3.873 \\
\hline $\begin{array}{l}\text { Barriers to effective management of lupus } \\
\text { due to social determinants of care in } \\
\text { predominantly lower socioeconomic status } \\
\text { areas }\end{array}$ & 6.937 \\
\hline $\begin{array}{l}\text { Lack of access to clinicians familiar with } \\
\text { lupus }\end{array}$ & 6.873 \\
\hline $\begin{array}{l}\text { Lack of access to medications either due to } \\
\text { lack of coverage or added cost to patients }\end{array}$ & 6.492 \\
\hline $\begin{array}{l}\text { Lack of understanding by government } \\
\text { Prioritisation Score }\end{array}$ \\
\hline
\end{tabular}

\section{Foundational barriers}

Clear findings emerged as pressing needs that are fundamental and necessary to address, before addressing other barriers. The known heterogeneity of lupus affects many other issues such as drug development, clinical care and access, and fully addressing this will take some time. For instance, the diversity of lupus manifestations means that two individuals with active disease may have non-overlapping manifestations. Addressing this using a single outcome measure therefore presents significant challenges. From a therapeutic perspective, identifying consistent subsets of patients at the molecular level will be needed to match the right targeted therapy to the correct patient group(s).

The high ranking of the development of biomarkers, whether prognostic, diagnostic or predictive, as an important challenge in lupus is not surprising as advances in this area will help accelerate drug development and movement towards precision medicine as well as more practical aspects of clinical care. Biomarkers can also provide value for clinical trials through disease stratification. ${ }^{18}$ While biomarker research has been prolific in the last decade and of top priority for clinical care and drug development, ${ }^{1920}$ it has not yet resulted in major breakthroughs. ${ }^{21}$ Researchers have suggested more concerted, longitudinal efforts across multiple study sites to identify and validate biomarker candidates, ${ }^{22}$ and the ALPHA project findings highlight that this has yet to be fully implemented.

The heterogeneity of lupus has also meant that many clinical trial designs may be fundamentally flawed. In addition, the lack of validated biomarkers to stratify and predict response limits progress and means many outcome measures remain suboptimal. Under-represented study populations, including paediatric populations, as well as common comorbidities, and a lack of adjustment in studies for non-adherence, all create limitations and challenges in current studies and trials. Any, or all, of these factors relate to patient and disease heterogeneity, which contribute to apparently 'unsuccessful' trials and, in particular, may lead to a drug which has efficacy not being taken forward in the drug development cycle. ${ }^{23}$ Recently in 2018, a panel of lupus experts, clinical trial investigators, biopharmaceutical developers and government stakeholders initiated an ongoing dialogue with the US Food and Drug Administration on optimising trials in part to accommodate for heterogeneity, ${ }^{24}$ and ALPHA results support addressing clinical trial design through synchronous efforts to bring more effective treatments to people with lupus.

\section{Lupus umbrella}

The ALPHA project also began to investigate how the lupus community views and categorises lupus as a spectrum, or umbrella, of related disorders. Lupus may coexist with and often presents with clinical features similar to other autoimmune diseases. ${ }^{25-28}$ Varying case definitions of lupus have been applied in epidemiological studies, ${ }^{29} 30$ which hinders the field's ability to produce consistent and comparable epidemiological data to further our understanding of the impact of lupus across populations. ${ }^{231}$ To address the spectrum concept, survey respondents were asked to select diseases from a list that they considered to be a part of the 'lupus' umbrella. Almost all respondents indicated lupus nephritis and cutaneous lupus, with a majority also selecting APS, mixed connective tissue disease and undifferentiated connective tissue disease. There were mixed opinions on numerous other diseases, supporting the need for further clarification of the 'lupus 
spectrum' and the disease definitions which may fall under this term to obtain and validate consensus.

\section{Feasibility}

The goal of the first phase of ALPHA was to identify and develop concrete and feasible strategies to address the fundamental challenges in lupus across drug development, clinical care and access. All of the mentioned challenges require large, concerted efforts among stakeholders who can lead the field and influence key decision makers with an agreed-on direction. Undoubtedly, substantial funds are needed to address top priorities and some issues may be more easily addressed than others. In the past decade, the overall funding landscape for lupus has been on a decline, ${ }^{32}$ particularly through the National Institutes of Health - the largest public funder of lupus research in the world- during a time in which arguably, lupus research has been prolific. Ongoing engagement of all lupus stakeholders is essential for addressing these barriers, and every stakeholder has a role, whether researcher, clinician, biopharmaceutical representative, regulatory official, insurer or patient.

\section{Limitations and strengths}

Limitations include potential bias among the respondent groups as the expert interview panel and survey used convenience samples. However, criteria were thoughtfully specified to identify a diverse group of lupus experts. There was also limited patient involvement in this first phase, as extensive understanding of the global lupus field was necessary to elicit and characterise fundamental challenges. However, both patient and patient advocacy representatives served on the GAC and were included in the interview stage. There may also be limitations in the survey instrument due to wording and question structure. For instance, under clinical care, 'lack of diagnostic, predictive and prognostic biomarkers for lupus' encompasses several different uses for biomarkers, so it was not possible to distinguish which specific use, if any, stakeholders considered most important. Additionally, the survey required forced-ranking among barriers in order to clearly identify challenges seen as top priorities to the field. All listed barriers were included in the survey based on concept elicitation from the expert interviews and thus all have unmet need. Therefore, low-ranking barriers are not synonymous with low importance. The overall response rate of $35 \%$ was not considered low, as it is consistent with typical responses rates among physician groups for web surveys. ${ }^{33}$

Particular strengths of this study include the global nature of this project and the engagement of a diverse set of lupus experts. There is international representation throughout all stages of this mixed-methods project, and the global survey received responses from individuals across 20 countries. Categories of stakeholders were also consistent for each stage of the project, and included lupus experts representing research, clinical practice, industry, government and patient advocacy in a variety of specialties.

\section{Future directions}

Phase II of this effort will include organising an international stakeholder meeting to develop a global road map of specific recommendations to address identified barriers, which may include multipronged strategies using regulatory and advocacy approaches, scientific consensus building, communication efforts, among other possible tactics. Special approaches may be needed to address issues specific to childhood lupus, as long-term outcomes and psychosocial impact of disease in children are likely different than in adults. Next steps include continued stakeholder engagement and further research efforts to characterise top barriers and lupus as a spectrum.

\section{CONCLUSION}

The ALPHA project has been organised as the first global, comprehensive initiative that addresses the entire spectrum of drug development, clinical care and access, and engages a global audience of lupus experts, including researchers, clinicians, industry and government representatives, patients and patient advocacy organisations. This initiative also explored views on lupus as a spectrum of related diseases. To date, failed clinical trials and variable access to care has led to to poor patient outcomes, high societal burden and excess mortality. Lupus is a major public health challenge that will require comprehensive measures to transform the research and healthcare landscape. Lupus experts must convene to determine feasible and coordinated approaches for addressing long-standing barriers across the global lupus community.

\section{Author affiliations}

${ }^{1}$ Medicine, Allegheny Health Network, Pittsburgh, Pennsylvania, USA

${ }^{2}$ Lupus Foundation of America Inc, Washington, District of Columbia, USA

${ }^{3}$ Tufts Center for the Study of Drug Development, Boston, Massachusetts, USA

${ }^{4}$ Lupus and Allied Diseases Association, Inc, Verona, New York, USA

${ }^{5}$ Rheumatology, Hanyang University Seoul Hospital, Seongdong-gu, , Korea (the

Republic of)

${ }^{6}$ Musculoskeletal and Dermatological Sciences, The University of Manchster,

Manchester, , UK

${ }^{7}$ Rheumatology and Clinical Immunology, Charite University Hospitals Berlin, Berlin, Germany

${ }^{8}$ EMD Serono Research and Development Institute, Billerica, Massachusetts, USA

${ }^{9}$ Rheumatology, Monash Medical Centre, Melbourne, Victoria, Australia

${ }^{10}$ Internal Medicine/Nephrology, Ohio State University Wexner Medical Center,

Columbus, Ohio, USA

${ }^{11}$ Pediatrics, Duke University, Durham, North Carolina, USA

${ }^{12}$ GlaxoSmithKline USA, Philadelphia, Pennsylvania, USA

${ }^{13}$ Dermatology, University of Pennsylvania, Philadelphia, Pennsylvania, USA

${ }^{14}$ Medicine, Brigham and Women's Hospital, Boston, Massachusetts, USA

Acknowledgements The authors thank all the individuals who participated in the expert interviews and in the global survey.

Contributors All authors contributed to the discussions and data interpretation contained in this paper and provided input on the manuscript and approved its final version. $A A$ and $Y P$ conducted expert interviews, survey programming and data analysis. KT and YP contributed to manuscript writing and finalising. $\mathrm{KG}$ and $\mathrm{LH}$ oversaw and approved all research efforts.

Funding The ALPHA Project was launched in partnership with founding partner EMD Serono Research \& Development, Inc. (a business of Merck KGaA, Darmstadt, Germany) and through additional support by GlaxoSmithKline. 
Competing interests IB is an NIHR Senior Investigator and is funded by Arthritis Research UK, the National Institute for Health Research Manchester Biomedical Research Unit and the NIHR/Wellcome Trust Manchester Clinical Research Facility. IB has received grants and/or honoraria and/or consultancy fees from Genzyme Sanofi, GSK, UCB, AstraZeneca, Medimmune, Eli Lilly, Merck Serono and ILT00. KC receives research support to study lupus from the NIH (R01 AR057327 and K24 AR066109), Merck and AstraZeneca. KC has received consulting honoraria $<\$ 5000$ from AstraZeneca. TD has received grants for study conduct and/or honoraria and /or consultancy fees from Janssen, GSK, Roche/Genentech, Eli Lilly, UCB, Merck Serono, AbbVie and Boston Pharmaceuticals. SM has received grants and/or honoraria and/or consultancy fees from Exagen Diagnostics, GSK, UCB and AstraZeneca. EM has received grants for study conduct and/or honoraria and/ or consultancy fees from AbbVie, Sanofi, GSK, UCB, AstraZeneca, Eli Lilly, Merck Serono and Janssen. BR has received grants and/or honoraria and/or consultancy fees from Aurinia, EMD Serono, Janssen and Medlmmune. BR is also a Principal Investigator on research projects for EMD Serono Research and Development Institute Inc. (Collaborated by Merck KGaA); Human Genome Sciences Inc, a GSK Company; NIH and RILITE Foundation. LES receives research support from CARRA and PCORI. LES is also on Data and Safety Monitoring Boards for UCB and Sanofi. VPW has received grants and/or honoraria from Celgene, Janssen, Biogen, Gilead, Viela, Medimmune, Pfizer, Resolve, AstraZeneca, Kyowa Hakko Kirin, Eli Lilly. Patient consent for publication Not required.

Provenance and peer review Not commissioned; externally peer reviewed. Data availability statement Data are available upon reasonable request.

Open access This is an open access article distributed in accordance with the Creative Commons Attribution Non Commercial (CC BY-NC 4.0) license, which permits others to distribute, remix, adapt, build upon this work non-commercially, and license their derivative works on different terms, provided the original work is properly cited, appropriate credit is given, any changes made indicated, and the use is non-commercial. See: http://creativecommons.org/licenses/by-nc/4.0/.

\section{REFERENCES}

1. Yen EY, Singh RR. Brief report: Lupus-An unrecognized leading cause of death in young females: a population-based study using nationwide death certificates, 2000-2015. Arthritis Rheumatol 2018;70:1251-5.

2. Stojan G, Petri M. Epidemiology of systemic lupus erythematosus: an update. Curr Opin Rheumatol 2018;30:144-50.

3. Singh RR, Yen EY. Sle mortality remains disproportionately high, despite improvements over the last decade. Lupus 2018;27:1577-81.

4. Carter EE, Barr SG, Clarke AE. The global burden of SLE: prevalence, health disparities and socioeconomic impact. Nat Rev Rheumatol 2016;12:605-20.

5. Agarwal N, Kumar V. Burden of lupus on work: issues in the employment of individuals with lupus. Work 2016;55:429-39.

6. Hersh AO, Case SM, Son MB, et al. Predictors of disability in a childhood-onset systemic lupus erythematosus cohort: results from the CARRA legacy registry. Lupus 2018;27:494-500.

7. Gillis JZ, Yazdany J, Trupin L, et al. Medicaid and access to care among persons with systemic lupus erythematosus. Arthritis Rheum 2007;57:601-7.

8. Demas KL, Costenbader KH. Disparities in lupus care and outcomes. Curr Opin Rheumatol 2009;21:102-9.

9. The Lewin Group Inc. Overcoming barriers to drug development in lupus, 2009. Available: https://www.lupus.org/s3fs-public/ documents/Overcoming_Barriers_to_Drug_Development_in_Lupus. pdf

10. Clinicaltrials.Gov, 2019. Available: https://clinicaltrials.gov/ [Accessed 21 Feb 2019].
11. Apostolopoulos D, Morand EF. It hasn't gone away: the problem of glucocorticoid use in lupus remains. Rheumatology 2016;24:kew406.

12. Tian J, Luo Y, Wu H, et al. Risk of adverse events from different drugs for SLE: a systematic review and network meta-analysis. Lupus Sci Med 2018;5:e000253.

13. Dall'Era M, Bruce I, Gordon C, et al. Current challenges in the development of new treatments for lupus. Ann Rheum Dis 2019.

14. Dolgin E. Lupus in crisis: as failures pile up, clinicians call for new tools. Nat Biotechnol 2019;37:7-8.

15. van Vollenhoven RF, Mosca M, Bertsias G, et al. Treat-to-target in systemic lupus erythematosus: recommendations from an international Task force. Ann Rheum Dis 2014;73:958-67.

16. van Vollenhoven R, Voskuyl A, Bertsias $G$, et al. A framework for remission in SLE: consensus findings from a large international Task force on definitions of remission in SLE (DORIS). Ann Rheum Dis 2017;76:554-61.

17. Franklyn K, Lau CS, Navarra SV, et al. Definition and initial validation of a lupus low disease activity state (LLDAS). Ann Rheum Dis 2016;75:1615-21.

18. Idborg H, Zandian A, Sandberg A-S, et al. Two subgroups in systemic lupus erythematosus with features of antiphospholipid or Sjögren's syndrome differ in molecular signatures and treatment perspectives. Arthritis Res Ther 2019;21.

19. Qi S, Chen Q, Xu D, et al. Clinical application of protein biomarkers in lupus erythematosus and lupus nephritis. Lupus 2018;27:1582-90.

20. Felten R, Sagez F, Gavand P-E, et al. 10 most important contemporary challenges in the management of SLE. Lupus Sci Med 2019;6:e000303.

21. Gensous N, Marti A, Barnetche T, et al. Predictive biological markers of systemic lupus erythematosus flares: a systematic literature review. Arthritis Res Ther 2017;19.

22. Liu C-C, Kao AH, Manzi S, et al. Biomarkers in systemic lupus erythematosus: challenges and prospects for the future. Ther Adv Musculoskelet Dis 2013;5:210-33.

23. Mahieu MA, Strand V, Simon LS, et al. A critical review of clinical trials in systemic lupus erythematosus. Lupus 2016;25:1122-40.

24. Merrill JT, Manzi S, Aranow C, et al. Lupus community panel proposals for optimising clinical trials: 2018. Lupus Sci Med 2018;5:e000258.

25. Lockshin MD, Levine AB, Erkan D. Patients with overlap autoimmune disease differ from those with 'pure' disease. Lupus Science \& Medicine 2015;2:e000084

26. Lorber M, Gershwin ME, Shoenfeld Y. The coexistence of systemic lupus erythematosus with other autoimmune diseases: the kaleidoscope of autoimmunity. Semin Arthritis Rheum 1994;24:105-13.

27. Jury EC, D'Cruz D, Morrow W. Autoantibodies and overlap syndromes in autoimmune rheumatic disease. J Clin Pathol 2001;54:340-7.

28. Merrill JT. Connective tissue diseases: is SLE many singleorgan diseases or an overlapping spectrum? Nat Rev Rheumatol 2015;11:385-6.

29. Dall'Era M, Cisternas MG, Snipes K, et al. The incidence and prevalence of systemic lupus erythematosus in San Francisco County, California: the California lupus surveillance project. Arthritis \& Rheumatology 2017;69:1996-2005.

30. Lim SS, Bayakly AR, Helmick CG, et al. The incidence and prevalence of systemic lupus erythematosus, 2002-2004: the Georgia lupus registry. Arthritis \& Rheumatology 2014;66:357-68.

31. Lim SS, Drenkard C. Epidemiology of lupus. Curr Opin Rheumatol 2015;27:427-32.

32. Estimates of funding for various research, condition, and disease categories (RCDC). Available: https://report.nih.gov/categorical_ spending.aspx [Accessed 4 Feb 2019].

33. Cunningham CT, Quan H, Hemmelgarn B, et al. Exploring physician specialist response rates to web-based surveys. BMC Med Res Methodol 2015;15. 